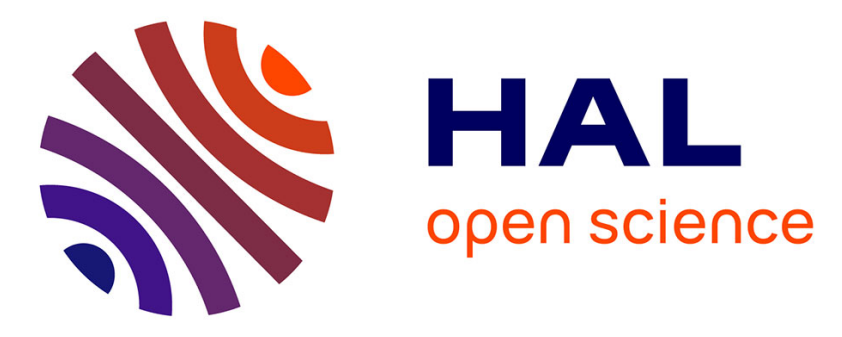

\title{
Prédiction des propriétés viscoélastiques des enrobés bitumineux Approches micromécaniques et expérimentales
}

Rabeb Cherif, Anissa Eddhahak, Thomas Gabet, Ferhat Hammoum, Jamel Néji

\section{To cite this version:}

Rabeb Cherif, Anissa Eddhahak, Thomas Gabet, Ferhat Hammoum, Jamel Néji. Prédiction des propriétés viscoélastiques des enrobés bitumineux Approches micromécaniques et expérimentales. Revue des composites et des matériaux avancés = Journal of Composite and Advanced Materials, 2017, 27 (1-2), pp.165-176. 10.3166/rcma.2017.00012 . hal-02547546

\section{HAL Id: hal-02547546 https://hal.science/hal-02547546}

Submitted on 20 Apr 2020

HAL is a multi-disciplinary open access archive for the deposit and dissemination of scientific research documents, whether they are published or not. The documents may come from teaching and research institutions in France or abroad, or from public or private research centers.
L'archive ouverte pluridisciplinaire HAL, est destinée au dépôt et à la diffusion de documents scientifiques de niveau recherche, publiés ou non, émanant des établissements d'enseignement et de recherche français ou étrangers, des laboratoires publics ou privés. 


\title{
Prédiction des propriétés viscoélastiques des enrobés bitumineux
}

\section{Approches micromécaniques et expérimentales}

\author{
Rabeb Cherif ${ }^{1}$, Anissa Eddhahak ${ }^{2}$, Thomas Gabet ${ }^{3}$, \\ Ferhat Hammoum $^{3}$, Jamel Neji ${ }^{1}$
}

1. Laboratoire de matériaux, d'optimisation et d'énergie pour la durabilité, École nationale d'ingénieurs de Tunis, BP 37, Le Belvédère, 1002, Tunis, Tunisie rabebcherif90@gmail.com, jamel.neji@enit.rnu.tn

2. PIMM, CNRS UMR 8006, arts et métiers ParisTech, 151, boulevard de l'Hôpital, 75013 Paris, France anissa.eddhahak@ensam.eu

3. IFSTTAR, MAST, MIT, route de Bouaye CS4, 44344 Bouguenais cedex, France thomas.gabet@ifsttar.fr,ferhat.hammoum@ifsttar.fr

RÉSUMÉ. Les enrobés bitumineux sont des mélanges de granulats et de bitume utilisés dans la construction de la majorité des réseaux routiers. Afin de préserver et garantir la durabilité des infrastructures routières, l'évaluation de la qualité et des performances de ces matériaux est indispensable. À ce titre, plusieurs recherches se sont focalisées sur le développement des modèles prédictifs, souvent empiriques, permettant de déduire les propriétés viscoélastiques d'un enrobé à partir de la connaissance des propriétés de ses constituants (bitume et/ou granulat). Dans ce contexte, nous proposons un modèle d'homogénéisation basé sur le schéma autocohérent généralisé (ACG) pour prédire le module complexe d'un enrobé à partir des propriétés de ses composants. Dans le but de valider le modèle proposé, plusieurs types d'enrobés (chauds et tièdes) fabriqués au laboratoire ont été testés, permettant ainsi la confrontation Expériencel Modélisation. Les résultats ont montré que l'on pouvait modéliser plusieurs types d'enrobés pour des températures inférieures à $20^{\circ} \mathrm{C}$. Cependant, au-delà de cette température, la précision du modèle diminue. Par ailleurs, la comparaison du modèle micromécanique aux autres modèles rhéologiques de la littérature a montré que le modèle proposé peut être tout aussi pertinent en termes de prédictions que les modèles rhéologiques considérés.

ABSTRACT. The asphalt mixtures are composed of aggregates and asphalt used in the construction of the majority of roads. In order to ensure the sustainability of the infrastructures, the evaluation of the quality and the performances of these materials are essential. In this context, several researches have been focused on the development of predictive models, often empirical ones, in order to deduce the viscoelastic properties of an asphalt mixture based on the properties of its constituents (binder and/or aggregate). In this context, we suggest a homogenization model based on the generalized self-consistent scheme (GSC) to predict the complex module of the asphalt concrete from the properties of its components. In the aim of the approach validation, different types of mixtures (hot and warm) made in the laboratory were tested. The results showed 
that one can predict the complex modulus of the different types of asphalt concretes for temperatures less than $20^{\circ} \mathrm{C}$. However, beyond this temperature, the precision of the model decreases. Besides, the comparison of the micromechanical model with the rheologic models in literature showed that the suggested model can be also relevant in terms of predictions as the considered models.

MOTS CLÉS : enrobé bitumineux, viscoélastique, homogénéisation, ACG, module complexe.

KEYWORDS: asphalt mixture, viscoelastic, homogenization, GSC, complex module.

\section{Extended abstract}

The evaluation of the quality and the performances of the asphalt mixtures are essential in order to ensure the sustainability of the infrastructures. In fact, the complex modulus testis one of the fundamental properties to check the viscoelastic performance of the asphalt mixtures. However, the laboratory measurement of complex modulus is a much time-consuming task, which requires carrying experiments at many scenarios of temperatures and frequencies solicitation, and trained staff to operate it, as well.

In this context, many researchers have been oriented to the determination of the viscoelastic properties, and notably the complex modulus, of asphalt mixture by using predictive approaches, empirical (Pouget et al., 2012, 2010; Bari et Witczak, 2006; Al-Khateeb et al., 2006; Christensen et al., 2003; Andrei et al., 1999) or micromechanical ones (Eddhahak-Ouni et al., 2015; Vu, 2014; Yin et al., 2008).

This paper presents the results of a homogenization model developed by the authors in a previous work (Eddhahak-Ouni et al., 2015) and a comparison with the experimental data. This model is based on the generalized self-consistent scheme (GSC) to predict the complex module of the asphalt concrete based on the properties of its components: binder, aggregate, filler, air void. In the aim of the approach validation, six types of mixtures were tested, four hot-mix asphalt (HMA1, HMA2, HMA3 and HMA4) and two warm-mix asphalt (WMA1 and WMA2). The results showed that one can predict the complex modulus of the different types of asphalt concretes for temperatures less than $20^{\circ} \mathrm{C}$. However, beyond this temperature, the precision of the model decreases.

In the aim of improving the results at high temperatures, the proposed model was compared with three rheological models of the literature; 2S2P1D (Pouget et al., 2012, 2010), Hirsh (Christensen et al., 2003) et Al-Khateeb (Al-Khateeb et al., 2006). Besides, the comparison of the micromechanical model with the rheological models in literature showed that the suggested model can be also relevant in terms of predictions as the considered models.

\section{Introduction}

Un enrobé est un mélange de granulats et de bitume qui constitue le plus souvent les couches supérieures d'une chaussée. La majorité des routes en Tunisie sont revêtues 
d'enrobés bitumineux. En outre, ce matériau a des propriétés assez complexes du fait du comportement viscoélastique du bitume qui le constitue. Pour garantir la durabilité des infrastructures, les performances des enrobés doivent être évaluées, ce qui nécessite un grand nombre d'essais expérimentaux. En particulier, la mesure du module complexe au laboratoire est coûteuse et peut durer plusieurs jours.

Dans ce contexte, plusieurs recherches se sont focalisées sur le développement des modèles plus rationnels permettant de prédire le module complexe d'un enrobé à partir des propriétés de ses constituants.

Certains modèles sont disponibles dans la littérature, parmi lesquels sont ceux empiriques ou rhéologiques (Pouget et al., 2012, 2010 ; Bari et Witczak, 2006 ; AlKhateeb et al., 2006 ; Christensen et al., 2003 ; Andrei et al., 1999 ) et d'autres basés sur des approches micromécaniques d'homogénéisation (Eddhahak-Ouni et al., 2015 ; Vu, 2014 ; Yin et al., 2008).

Ce travail propose un modèle micromécanique basé sur le schéma auto-cohérent généralisé (ACG) permettant de prédire le module complexe du béton bitumineux. Dans le but de valider ce modèle, différents types d'enrobés ont été testés et les modules complexes expérimentaux ont été comparés avec ceux obtenus numériquement. Le modèle proposé a été comparé à d'autres modèles empiriques connus de la littérature (Pouget et al., 2012, 2010 ; Al-Khateeb et al., 2006 ; Christensen et al., 2003) et la comparaison a montré qu'il est aussi pertinent en termes de prédiction que les modèles rhéologiques considérés.

\section{Présentation des modèles}

Cette partie est consacrée à la présentation des modèles prédictifs, à savoir le modèle d'homogénéisation développé dans les travaux antérieurs des auteurs (Eddhahak-Ouni et al., 2015) et les modèles rhéologiques qui seront considérés pour la comparaison. Ces modèles ont tous pour objectif de prédire le comportement d'un enrobé bitumineux à partir de la connaissance des propriétés de ses constituants. Le modèle micromécanique proposé se distingue par sa prise en compte de la microstructure induite par le processus de fabrication, en plus des propriétés des constituants, pour la prédiction des propriétés équivalentes du matériau hétérogène. À noter que l'effet « microstructure » ne fait pas l'objet de la présente étude.

\subsection{Modèle micromécanique}

L'enrobé bitumineux peut être modélisé comme étant un matériau multiphasique constitué d'une phase inclusionnaire que représentent les granulats, d'une phase matrice donnée par le mastic (mélange « bitume + fines ») et d'une phase poreuse qui représente le vide interganulaire (figure 1). Dans cette étude, le schéma auto-cohérent généralisé (Hervé et Zaoui, 1993 ; Christensen et Lo, 1979) a été retenu pour décrire le comportement équivalent du matériau hétérogène. 


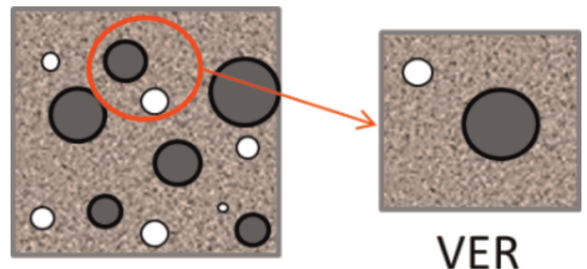

Figure 1. Modélisation schématique de l'enrobé bitumineux

Les phases sont caractérisées par leurs modules de cisaillement $\mu_{\mathrm{i}}$, leurs modules de compressibilités $k_{\mathrm{i}}$ et leurs fractions volumiques $f_{\mathrm{i}}$ dans le mélange.

L'homogénéisation du problème global noté « $\mathrm{GP}$ » peut être réalisée en considérant simultanément deux sous-problèmes notés «SP1 » et «SP2 » associés respectivement à l'inclusion rigide (les granulats) et les vides soumis à la même déformation $E_{0}$ à l'infini comme le montre la figure 2. Ainsi, toute la difficulté réside dans la résolution d'un problème de localisation global qui permet de trouver le tenseur reliant les propriétés macroscopiques de l'enrobé aux propriétés microscopiques.

Ainsi, le module complexe homogénéisé noté $\mu^{\text {hom }}$ peut être obtenu tel que :

$$
\mu^{\text {hom }}=\langle\mu: A\rangle=\sum f_{i} \mu_{i}:\left\langle A_{i}\right\rangle,
$$

où $\mu^{\text {hom }}$ est le tenseur de rigidité homogénéisé de $4^{\mathrm{e}}$ ordre, $A_{\mathrm{i}}$ est le tenseur de localisation de déformations de $4^{\mathrm{e}}$ ordre associé au sous problème $P_{\mathrm{i}}(i=1$ ou 2$)$.

Dans le cas du problème général, le tenseur de localisation des déformations noté $L$ relie la déformation microscopique $\varepsilon$ et celles macroscopique $E$ imposée à l'infini :

$$
\varepsilon_{i}(x)=\left\langle L_{i}(x)\right\rangle: E
$$

Le tenseur de localisation des déformations du problème global est lié au problème secondaire par la relation suivante :

$$
\left\langle L_{i}(x)\right\rangle=\left\langle A_{i}\right\rangle:\left(\sum f_{\mathrm{i}}\left\langle A_{\mathrm{i}}\right\rangle\right)^{-1}
$$

Dans le cas linéaire et isotrope, le tenseur de rigidité est donné par :

$$
C=3 k I^{\mathrm{sph}}+2 \mu I^{\mathrm{dev}}
$$

où $I^{\mathrm{sh}}$ et $I^{\mathrm{dev}}$ sont respectivement les opérateurs sphériques et déviatoriques.

Une fois les tenseurs de localisation des problèmes auxiliaires déterminés, on peut déduire le tenseur de localisation du problème global. Enfin, nous obtenons par la suite 


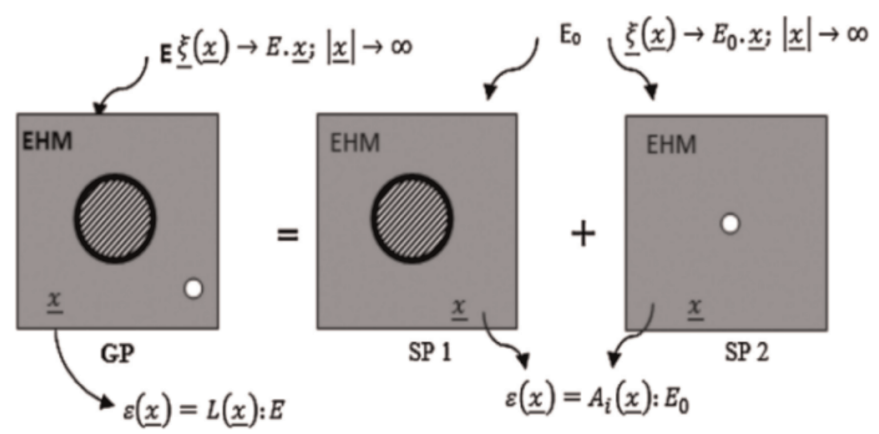

Figure 2. Schéma du problème global d'homogénéisation «GP» (Eddhahak-Ouni et al., 2015)

les modules de compressibilité et de cisaillement homogénéisés :

$$
\left\{\begin{array}{l}
K^{h o m}=\left(f_{\mathrm{a}} k_{\mathrm{a}} A_{\mathrm{a}}^{\mathrm{sph}}+f_{\mathrm{m}} k_{\mathrm{m}} A_{\mathrm{m}}^{\mathrm{sph}}+f_{\mathrm{p}} k_{\mathrm{p}} A_{\mathrm{p}}^{\mathrm{sph}}\right):\left(f_{\mathrm{a}} A_{\mathrm{a}}^{\mathrm{sph}}+f_{\mathrm{m}} A_{\mathrm{m}}^{\mathrm{sph}}+f_{\mathrm{p}} A_{\mathrm{p}}^{\mathrm{sph}}\right)^{-1} \\
\mu^{h o m}=\left(f_{\mathrm{a}} \mu_{\alpha} A_{\mathrm{a}}^{\mathrm{dev}}+f_{\mathrm{m}} \mu_{\mathrm{m}} A_{\mathrm{m}}^{\mathrm{dev}}+f_{\mathrm{p}} \mu_{\mathrm{p}} A_{\mathrm{p}}^{\mathrm{dev}}\right):\left(f_{\mathrm{a}} A_{\mathrm{a}}^{\mathrm{dev}}+f_{\mathrm{m}} A_{\mathrm{m}}^{\mathrm{dev}}+f_{\mathrm{p}} A_{\mathrm{p}}^{\mathrm{dev}}\right)^{-1}
\end{array}\right.
$$

Par la suite, et en supposant que le comportement est linéaire et isotrope, on peut déduire la norme du module complexe :

$$
E^{\text {hom }}=\frac{9 k^{\text {hom }} \mu^{\text {hom }}}{3 k^{\text {hom }}+\mu^{\text {hom }}} .
$$

La figure 3 présente un digramme simplifié du modèle micro-macro résumant les différentes données du problème en passant par le schéma auto-cohérent généralisé afin d'obtenir le module complexe équivalent de l'enrobé bitumineux.

Dans la suite, on présentera trois modèles rhéologiques qui seront considérés pour l'évaluation de la pertinence du modèle d'homogénéisation présenté.

\subsection{Modèle de Hirsch}

Le modèle de Hirsch est basé sur la loi de mélanges des matériaux composites (Christensen et al., 2003). Cette loi de mélange a été développée par Hirsch vers les années 1960. La disposition des différentes phases du matériau est considérée selon des schémas en parallèle ou en série.

Ce modèle permet ainsi la prédiction du module d'un matériau composite par une loi en série ou en parallèle, selon l'équation suivante : 


\section{Input}

-Module de compressibilité et de cisaillement de bitume pour chaque température et fréquence.

-Module de compressibilité et de cisaillement des granulats et des fillers.

-Fractions volumiques des différents constituants.

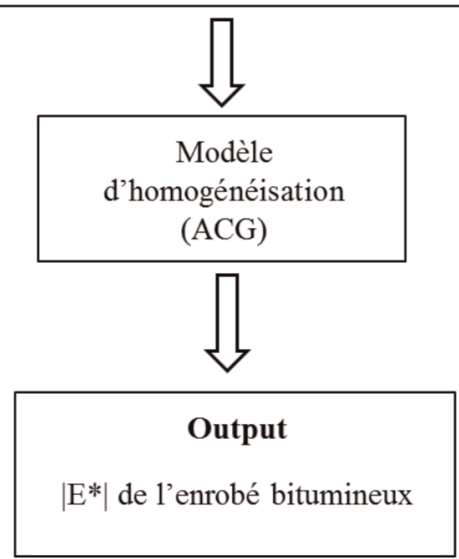

Figure 3. Étapes du modèle micromécanique proposé

$$
\begin{aligned}
\left|E^{*}\right|_{m}= & P_{c}\left[4200000\left(1-\frac{V M A}{100}\right)+3\left|G^{*}\right|_{\mathrm{b}}\left(\frac{(V F A * V M A)}{10000}\right)\right] \\
& +\frac{\left(1-P_{c}\right)}{\frac{\left(1-\frac{V M A}{100}\right)}{4200000}+\left(\frac{V M A}{3\left|G^{*}\right|_{b} V F A}\right)}
\end{aligned}
$$

avec :

$$
\begin{gathered}
P_{c}=\frac{\left[20+\frac{33\left|G^{*}\right|_{b} V F A}{V M A}\right]^{0,58}}{650+\left(\frac{3\left|G^{*}\right|_{b} V F A}{V M A}\right)}, \\
V M A=\frac{100-M V A_{b} x\left(100-T_{\mathrm{b}}\right)}{M V A_{g}}, \\
V F A=\frac{V M A-V}{V M A} \times 100,
\end{gathered}
$$


où :

- $\left|E^{*}\right|_{\mathrm{m}}$ : le module complexe de l'enrobé ;

- $\left|G^{*}\right|_{\mathrm{b}}$ : le module de cisaillement de bitume ;

- $M V A_{\mathrm{b}}$ : la masse volumique apparente de bitume ;

- $M V A_{\mathrm{g}}$ : la masse volumique apparente des granulats ;

- $V$ : pourcentage de vide ;

$-T_{\mathrm{b}}:$ teneur en bitume.

\subsection{Modèle d'Al-Khateeb}

Le modèle d'Al-Khateeb est un modèle basé sur le modèle de Hirsch. Il permet de prédire la norme de module complexe de l'enrobé selon la relation suivante (Al-Khateeb et al., 2006) :

$$
\left|E^{*}\right|_{m}=3\left[\frac{100-V M A}{100}\right]\left[\frac{90+10000 *\left(\frac{\left|G^{*}\right|_{b}}{V M A}\right)^{0,66}}{1100+\left(900 * \frac{\left|G^{*}\right|_{b}^{0,66}}{V M A}\right)}\right]\left|G^{*}\right|_{g}
$$

où :

- $\left|E^{*}\right|_{\mathrm{m}}$ : le module complexe de l'enrobé ;

- $\left|G^{*}\right|_{\mathrm{b}}$ : le module de cisaillement de bitume ;

- $\left|G^{*}\right|_{\mathrm{g}}$ : le module de cisaillement de bitume à l'infini.

\subsection{Modèle 2S2P1D}

Ce modèle a été développé par Di Benedetto et al. selon l'équation (12) :

$$
E^{*}(\omega, T)=E_{0, \text { mix }}+\left[E^{*}\left(10^{\alpha} \omega, T\right)-E_{0, \text { binder }}\right] \times\left[\frac{E_{00, \text { mix }}-E_{0, \text { mix }}}{E_{00, \text { binder }}-E_{0, \text { binder }}}\right],
$$

où :

- $E_{\text {mix }}^{*}$ : le module complexe de l'enrobé ;

- $E_{\text {binder }}^{*}$ : le module complexe de bitume ;

$-E_{0, \text { mix }}^{*}$ : valeurs asymptotiques minimales du module complexe de l'enrobé ;

- $E_{0, \text { binder }}^{*}$ valeurs asymptotiques minimales du module complexe bitume ;

- $E^{*}$ 00mix valeurs asymptotiques maximales du module complexe de l'enrobé ;

$-E^{*}{ }_{00, \text { binder }}$ valeurs asymptotiques maximales du module complexe du bitume. 
Le paramètre supplémentaire $\alpha$ dépend de la formulation d'enrobé et du vieillissement durant l'enrobage (Pouget et al., 2012, 2010).

$$
\tau_{0, \text { mix }}=10^{\alpha} \tau_{0, \text { binder }} .
$$

\section{Matériaux testés}

Afin de valider le modèle micromécanique, différents types d'enrobés ont été fabriqués et testés au laboratoire permettant ainsi la confrontation Expérience/ Modélisation.

Six types d'enrobés ont été testés, quatre fabriqués avec une technique de malaxage à chaud (HMA1, HMA2, HMA3 et HMA4) et deux fabriqués avec une technique tiède (WMA1 et WMA2) définis ainsi :

- HMA1 : enrobé français ; BBSG 0/10, contenant 50 \% de fraisâts (Lopes et al., 2014) ;

- HMA2 : enrobé français ; BBSG 0/10 (Lopes et al., 2014);

- HMA3 : enrobé français ; BBSG 0/14, contenant $15 \%$ de fraisâts ;

- HMA4 : enrobé français ; BBSG 0/14 (Alam, 2015) ;

- WMA1 : enrobé français ; BBSG 0/10, contenant $50 \%$ de fraisâts (Lopes et al., 2014) ;

- WMA2 : enrobé français ; BBSG 0/10 (Lopes et al., 2014).

Les essais expérimentaux ont été réalisés à l'Institut français des sciences et technologies des transports, de l'aménagement et des réseaux (IFSTTAR-Nantes) dans le cadre des travaux de recherche (Lopes et al., 2014 ; Alam, 2015).

\section{Résultats et discussion}

La figure 4 présente la comparaison entre le module complexe expérimental et celui obtenu par le modèle d'homogénéisation proposé dans le cas des six enrobés testés au laboratoire. Les résultats montrent que le modèle donne une bonne prédiction du module complexe pour les différents types d'enrobés étudiés.

La comparaison des courbes maîtresses expérimentales et numériques présentées sur la figure 5 montre que le modèle d'homogénéisation donne des résultats très proches des résultats expérimentaux.

Sur la figure 6, nous comparons les modules complexes numériques avec les modules expérimentaux dans le cas de l'enrobé HMA3. On peut constater d'après les courbes présentées que les résultats sont en bonne concordance pour les températures inférieures à $20^{\circ} \mathrm{C}$ pour toute la gamme de fréquences allant de $1 \mathrm{~Hz}$ à $40 \mathrm{~Hz}$. Cependant, à partir de $20^{\circ} \mathrm{C}$ la précision du modèle diminue. 


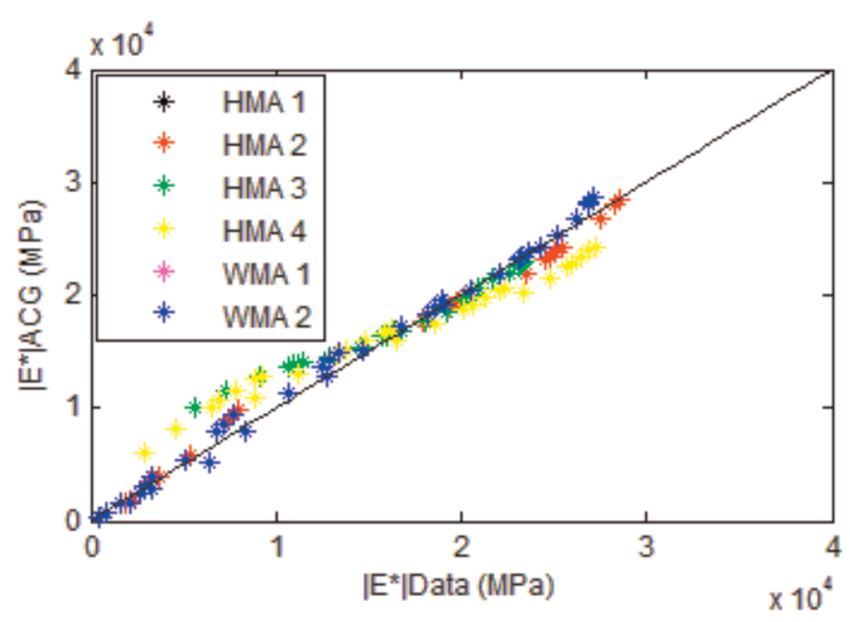

Figure 4. Module complexe $\left|E^{*}\right|$ donné par l'expérience et le modèle micro-macro à $T=15^{\circ} \mathrm{C}$

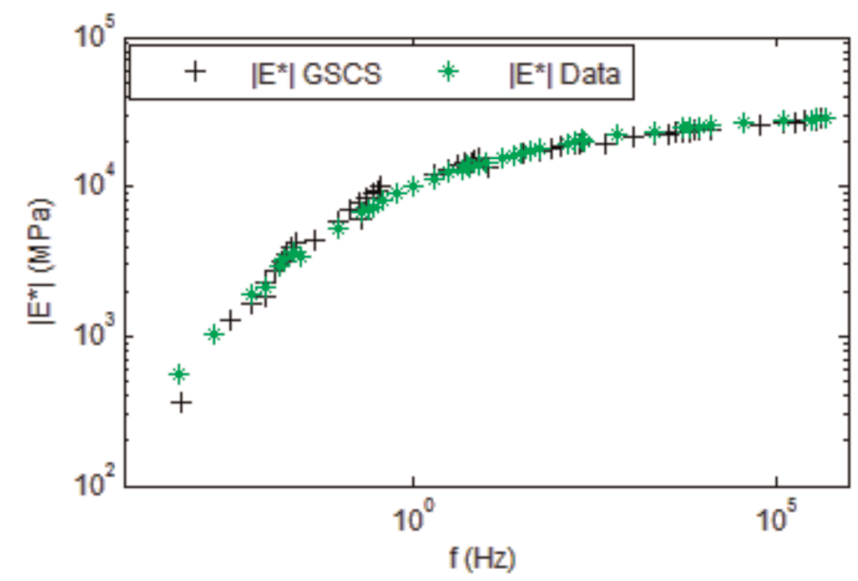

Figure 5. Courbes maîtresses expérimentale et numérique de l'enrobé HMA1 à $T=15^{\circ} \mathrm{C}$

La figure 7 illustre les résultats de comparaison des courbes maîtresses expérimentales avec les courbes maîtresses obtenues numériquement. On remarque que tous les modèles présentent des tendances similaires avec des approximations plutôt satisfaisantes dans l'ensemble. Par ailleurs, le modèle d'homogénéisation semble aussi pertinent en termes de prédiction que les modèles rhéologiques considérés. 

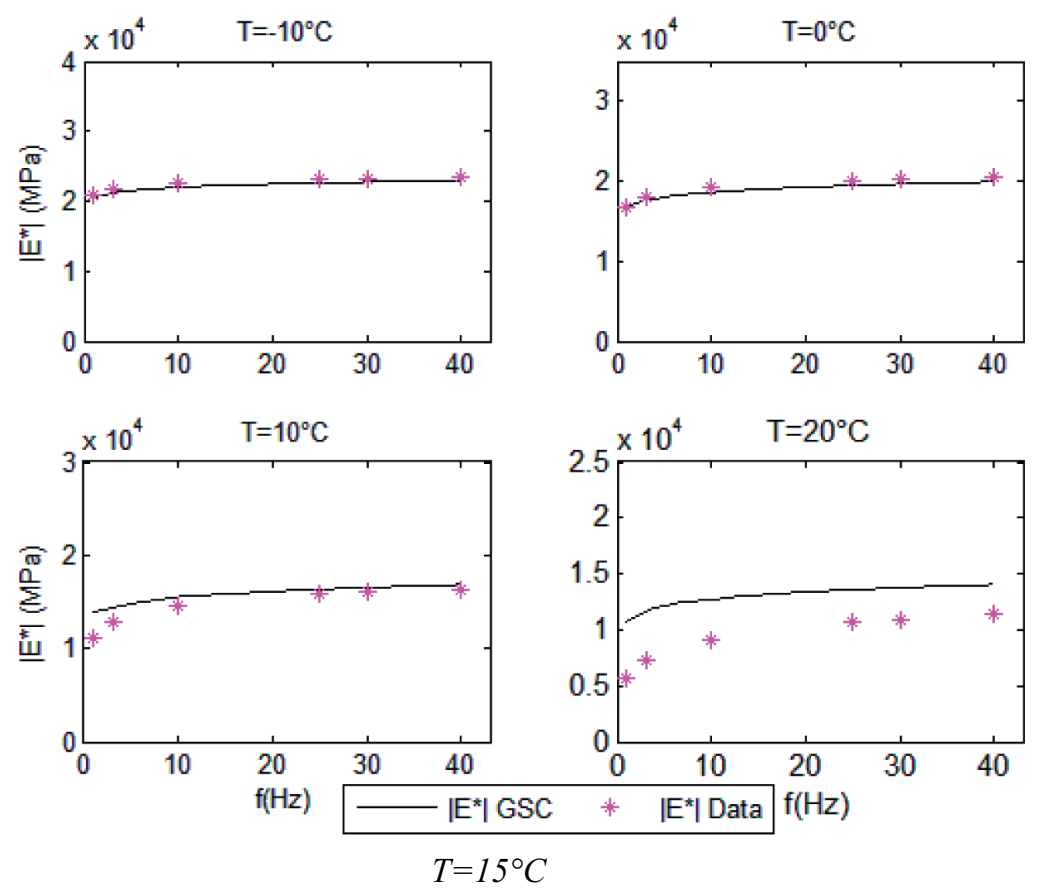

Figure 6. Comparaison du module complexe expérimental et numérique à différentes températures (cas HMA3)

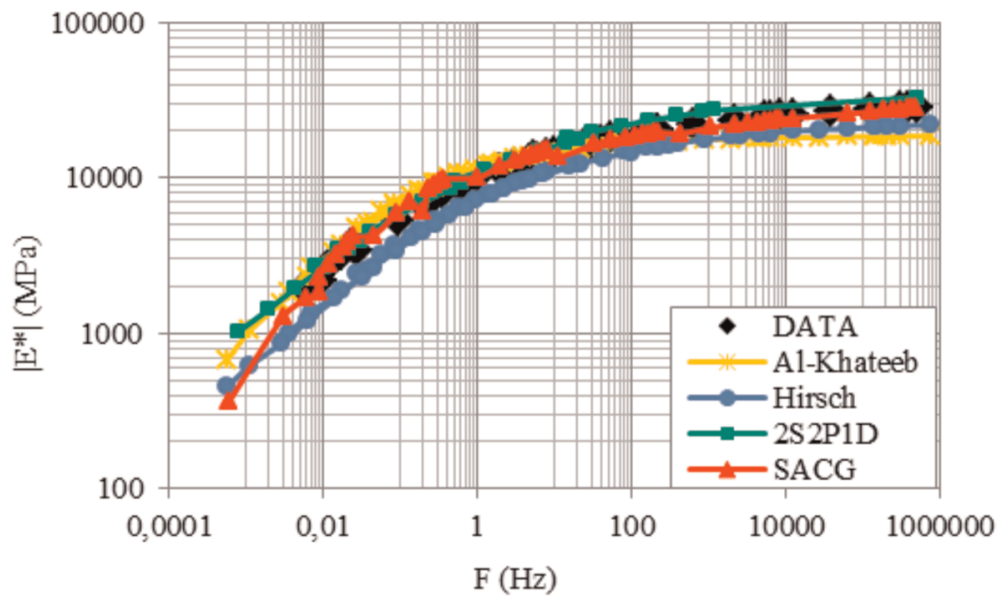

Figure 7. Courbes maîtresses issues de l'expérience et des modèles prédictifs 


\section{Conclusion}

Dans cette étude, un modèle d'homogénéisation basé sur le schéma auto-cohérent généralisé a été proposé et testé sur différents types d'enrobés, afin de prédire le comportement effectif de l'enrobé à partir des propriétés de ses constituants. Le modèle proposé a été comparé à d'autres modèles rhéologiques de la littérature dans le but d'améliorer les résultats à hautes températures. Par ailleurs, la comparaison du modèle micromécanique avec des modèles rhéologiques prédictifs très connus a montré que le modèle proposé est très satisfaisant pour la prédiction du module complexe des enrobés bitumineux chauds et tièdes, et ce pour des températures inférieures à $20^{\circ} \mathrm{C}$. Des améliorations du modèle s'avèrent néanmoins nécessaires afin d'avoir de meilleures estimations du module équivalent à plus hautes températures.

\section{Bibliographie}

Alam S.Y. (2015). Détermination des propriétés mécaniques d'enrobes bitumineux à partir des caractéristiques des constituants. Rapport de recherche, laboratoire central des Ponts et Chaussées, université de Marne la Vallée.

Al-Khateeb G., Shenoy A., Gibson N., Harman T. (2006). A new simplistic model for dynamic modulus predictions of asphalt paving mixtures. J. Assoc. Asph. Paving Technol., vol. 75, p. 1254-1293.

Andrei D., Witczak M.W., Mirza M.W. (1999). Development of a revised predictive model for the dynamic (complex) modulus of asphalt mixtures. NCHRP 1-37A Interim Team Report University of Maryland.

Bari J., Witczak M.W. (2006). Development of a new revised version of the Witczak E* predictive model for hot mix asphalt mixtures. J. Assoc. Asph. Paving Technol., vol. 75, p. 381-423.

Christensen R.M., Lo K.H. (1979). Solutions for effective shear properties in three phase sphere and cylinder models. J. Mech. Phys. Solids, vol. 27, p. 315-330.

Christensen D.W., Pellinen T., Bonaquist R.F. (2003). Hirsch model for estimating the modulus of asphalt concrete. J. Assoc. Asph. Paving Technol., vol. 72, p. 97-121.

Eddhahak-Ouni A., Vandamme M., Vu V.T. (2015). Micromechanical contribution for the prediction of the viscoelastic properties of high rate recycled asphalt and influence of the level blending. Arch. Civil Mech. Eng., vol. 312.

Hervé E., Zaoui A. (1993). N-Layered inclusion based micromechanical modeling. Int. J. Eng. Sci., vol. 31, p. 1-10.

Lopes M., Gabet T., Bernucci L., Mouillet V., Brosseaud Y. (2014). Coupling WMA technique to a high rate of RAP, in laboratory. Mater. Struct., doi: 10.1617/s11527-014- 0454-9.

Pouget S., Sauzéat C., Di Benedetto H., Olard F. (2010). From the behavior of constituent materials to the calculation and design of orthotropic bridge structures. Road Mater. Pavement Des., vol. 11, no 1, p. 111-144. doi: 10.1080/14680629.2010.9690329. 
Pouget S., Sauzéat C., Di Benedetto H., Olard F. (2012). Modeling of viscous bituminous wearing course materials on orthotropic steel deck. Mater. Struct., vol. 45, p. 1115-1127, doi: 10.1617/ s11527-011-9820-z.

$\mathrm{Vu}$ V.T. (2014). Modélisation multi-échelle du comportement viscoélastique de l'enrobé bitumineux a fort recyclage : approche numérique. Rapport de recherche ESTP.

Yin H.M., Buttlar W.G., Paulino G.H., Di Benedetto H. (2008). Assessment of existing micromechanical models for asphalt mastics considering viscoelastic effects. Road Mater. Pavement Des., vol. 9, n 1, p. 31-57. 